\title{
Gauge freedom for degenerate Bloch states
}

\author{
F. Pientka, ${ }^{1,2, *}$ M. Gradhand, ${ }^{3,4}$ D. V. Fedorov, ${ }^{2,4}$ I. Mertig, ${ }^{2,4}$ and B. L. Györffy ${ }^{3}$ \\ ${ }^{1}$ Dahlem Center for Complex Quantum Systems and Fachbereich Physik, Freie Universität Berlin, D-14195 Berlin, Germany \\ ${ }^{2}$ Institute of Physics, Martin Luther University Halle-Wittenberg, D-06099 Halle, Germany \\ ${ }^{3}$ H.H. Wills Physics Laboratory, University of Bristol, Bristol BS8 1TH, United Kingdom \\ ${ }^{4}$ Max Planck Institute of Microstructure Physics, Weinberg 2, D-06120 Halle, Germany
}

(Received 2 February 2012; revised manuscript received 16 May 2012; published 9 August 2012)

\begin{abstract}
In nonmagnetic crystals with inversion symmetry the electronic bands are twofold degenerate. As a consequence, any orthonormalized linear combination of the two corresponding eigenfunctions can represent the electron wave function. A priori it is not obvious which superposition, gauge, should be chosen to calculate a quantity which is not gauge invariant within a certain approximation. Here we consider gauge options appropriate under particular physical conditions.
\end{abstract}

DOI: 10.1103/PhysRevB.86.054413

PACS number(s): 71.15.-m, 71.20.-b, 75.70.Tj

According to Kramers theorem, ${ }^{1}$ the presence of both space and time inversion symmetry makes each band in a crystal double degenerate. ${ }^{2}$ In a nonrelativistic theory the two degenerate subbands consist of pure "spin-up" $\left|\Psi_{n \mathbf{k}}^{\uparrow}\right\rangle$ or "spin-down" $\left|\Psi_{n \mathbf{k}}^{\downarrow}\right\rangle$ states, where $\mathbf{k}$ is the wave vector and $n$ is the band index. In the presence of spin-orbit coupling the two subbands are still degenerate, but they cannot simply be labeled by the spin since it is no longer a good quantum number. The motivation of this paper is to highlight some consequences of this degeneracy arising in practical calculations for spin and charge transport in nonmagnetic solids. Hereafter we will call a pair of two degenerate states a Kramers doublet.

In the semiclassical theory one constructs wave packets out of Bloch states and discusses the dynamics of such wave packets in real and reciprocal space. In order to describe the time evolution of a wave packet correctly, the effect of the Berry phase should be taken into account via the Berry curvature. ${ }^{3-6}$ If two or more eigenstates are degenerate, adiabatic transitions between different members of the degenerate subspace may occur. Then, the effect of the Berry phase must be extended to an $\mathrm{SU}(N)$ gauge theory, ${ }^{7,8}$ where $N$ is the dimension of the degenerate subspace. Consequently, the Berry curvature should be promoted to have a matrix form. Of course, in a proper theory observables must be invariant with respect to any transformation within the degenerate subspace represented by a unitary $N \times N$ matrix.

Nevertheless, an approximate treatment based on the singlesubband picture and used for nonmagnetic and inversion symmetric crystals can provide good agreement with the fully quantum mechanical approach ${ }^{9}$ as well as with experiment. ${ }^{10}$ However, for practical calculations within such an approximation one needs to choose appropriate conditions for fixing the unitary transformation mentioned above. In other words, a global gauge valid for all points in the momentum space is required. Here we consider two gauges which can be used in practice. Both of them are inspired by the spin polarization, the expectation value of the spin operator, in the nonrelativistic limit. In the first one, which we will call gauge I, the spin polarization is chosen to be parallel for all $\mathbf{k}$ states. In the second gauge, gauge II, one component of the spin operator is diagonalized with respect to the two degenerate states discussed above.
In what follows, we show that the most appropriate choice is gauge II. In this gauge we avoid points in $\mathbf{k}$ space with a vanishing spin polarization and a discontinuous Berry curvature arising as an artifact of gauge I. We will illustrate this fact by tight-binding model calculations. ${ }^{11}$ Then, we compare results for the spin relaxation time and the spin Hall angle of the extrinsic spin Hall effect obtained with the two gauges using a Korringa-Kohn-Rostoker (KKR)-based approach. ${ }^{10,12,13}$ Finally, we show that even beyond the singlesubband approximation gauge II is still attractive to be applied for the simplification of calculations of the intrinsic spin Hall conductivity.

For a nondegenerate Bloch state of the form $\left\langle\mathbf{r} \mid \Psi_{n \mathbf{k}}\right\rangle=$ $e^{i \mathbf{k} \cdot \mathbf{r}} u_{n \mathbf{k}}(\mathbf{r})$, where the periodic part $u_{n \mathbf{k}}(\mathbf{r})$ is generally a spinor, the Berry curvature is defined as ${ }^{3,6}$

$$
\boldsymbol{\Omega}_{n}(\mathbf{k})=i\left\langle\nabla_{\mathbf{k}} u_{n \mathbf{k}}|\times| \nabla_{\mathbf{k}} u_{n \mathbf{k}}\right\rangle .
$$

The function $u_{n \mathbf{k}}$ is defined only up to a phase factor and the corresponding gauge transformation leaves the Berry curvature invariant. Since the gauge group is $U(1)$, one can call this curvature Abelian. Here we are interested in the more complex non-Abelian case, ${ }^{7,8}$ where the gauge transformation is a unitary operator in the Hilbert space spanned by the two degenerate states forming a Kramers doublet at each $\mathbf{k}$ point in the Brillouin zone (BZ).

Let us label the above two states as "+" and "-," so far in arbitrary order. Then, the Berry curvature can be represented as an $\mathfrak{s u}(2)$ vector-valued matrix ${ }^{6}$

$$
\hat{\mathbf{\Omega}}_{\mathbf{k}}=\left(\begin{array}{ll}
\boldsymbol{\Omega}_{++}(\mathbf{k}) & \boldsymbol{\Omega}_{+-}(\mathbf{k}) \\
\boldsymbol{\Omega}_{-+}(\mathbf{k}) & \boldsymbol{\Omega}_{--}(\mathbf{k})
\end{array}\right),
$$

with the matrix elements being the following vectors:

$$
\begin{aligned}
\boldsymbol{\Omega}_{s s^{\prime}}(\mathbf{k})= & i\left\{\left\langle\nabla_{\mathbf{k}} u_{\mathbf{k}}^{s}|\times| \nabla_{\mathbf{k}} u_{\mathbf{k}}^{s^{\prime}}\right\rangle-\left\langle\nabla_{\mathbf{k}} u_{\mathbf{k}}^{s} \mid u_{\mathbf{k}}^{+}\right\rangle \times\left\langle u_{\mathbf{k}}^{+} \mid \nabla_{\mathbf{k}} u_{\mathbf{k}}^{s^{\prime}}\right\rangle\right. \\
& \left.-\left\langle\nabla_{\mathbf{k}} u_{\mathbf{k}}^{s} \mid u_{\mathbf{k}}^{-}\right\rangle \times\left\langle u_{\mathbf{k}}^{-} \mid \nabla_{\mathbf{k}} u_{\mathbf{k}}^{s^{\prime}}\right\rangle\right\},
\end{aligned}
$$

where $s, s^{\prime} \in\{+,-\}$. The non-Abelian Berry curvature is gauge covariant and hence its matrix elements are not observable. However, such quantities as $\operatorname{Tr}\left[\hat{\mathbf{\Omega}}_{\mathbf{k}}\right]$ or $\operatorname{det}\left|\hat{\mathbf{\Omega}}_{\mathbf{k}}\right|$ are gauge invariant. In the case of the considered Kramers doublets, the trace vanishes at each $\mathbf{k}$ point due to $\boldsymbol{\Omega}_{--}(\mathbf{k})=-\boldsymbol{\Omega}_{++}(\mathbf{k})$ and only the determinant remains finite. It may be used to visualize the Berry curvature as a function of the crystal momentum. 
On the other hand, within the semiclassical theory one may be interested in a global gauge, where the diagonal components of the Berry curvature can be visualized as meaningful quantities, in order to consider contributions from individual subbands.

A quite reasonable choice of a gauge is to ensure that the spin polarization of the two degenerate states is always parallel and antiparallel to the $z$ direction chosen as a global quantization axis. Here we define the spin polarization as the expectation value of the spin operator $\hat{\mathbf{S}}=\left(\hat{S}_{x} ; \hat{S}_{y} ; \hat{S}_{z}\right)$ expressed in units of $\hbar / 2$. This operator is represented by $\hat{\mathbf{S}}=$ $\hat{\boldsymbol{\sigma}}$ or $\hat{\mathbf{S}}=\hat{\beta} \hat{\boldsymbol{\sigma}}$ for the Pauli or the Dirac spinors, respectively. In addition, we can connect the labels "+" and "-," which were introduced above for the two degenerate states, to the sign of their $z$ component of the spin polarization. Such a gauge, let us call it gauge $I$, requires ${ }^{14}$

$$
\begin{aligned}
& \left\langle\Psi_{\mathrm{I}}^{+}\left|\hat{S}_{x}\right| \Psi_{\mathrm{I}}^{+}\right\rangle=\left\langle\Psi_{\mathrm{I}}^{-}\left|\hat{S}_{x}\right| \Psi_{\mathrm{I}}^{-}\right\rangle=0, \\
& \left\langle\Psi_{\mathrm{I}}^{+}\left|\hat{S}_{y}\right| \Psi_{\mathrm{I}}^{+}\right\rangle=\left\langle\Psi_{\mathrm{I}}^{-}\left|\hat{S}_{y}\right| \Psi_{\mathrm{I}}^{-}\right\rangle=0, \\
& \left\langle\Psi_{\mathrm{I}}^{+}\left|\hat{S}_{z}\right| \Psi_{\mathrm{I}}^{+}\right\rangle=-\left\langle\Psi_{\mathrm{I}}^{-}\left|\hat{S}_{z}\right| \Psi_{\mathrm{I}}^{-}\right\rangle \geqslant 0,
\end{aligned}
$$

which fixes the two free parameters of the corresponding unitary transformation. ${ }^{15}$ The identity in the last line of Eq. (4) holds for all components of the spin operator

$$
\left\langle\Psi_{\mathbf{k}}^{+}|\hat{\mathbf{S}}| \Psi_{\mathbf{k}}^{+}\right\rangle=-\left\langle\Psi_{\mathbf{k}}^{-}|\hat{\mathbf{S}}| \Psi_{\mathbf{k}}^{-}\right\rangle
$$

which can be shown by using the relation of the two Kramers states $\left|\Psi_{\mathbf{k}}^{-}\right\rangle=\hat{\mathrm{I}} \hat{\mathrm{T}}\left|\Psi_{\mathbf{k}}^{+}\right\rangle$, where $\hat{\mathrm{I}}$ and $\hat{\mathrm{T}}$ are the space-inversion and time-reversal operators. ${ }^{1,2}$ The gauge given by Eq. (4) is inspired by the nonrelativistic picture where the spin on its own is a good quantum number pointing along or opposite to the global quantization axis.

Similarly to the non-Abelian Berry curvature, one can treat the spin polarization more generally as an $\mathfrak{s u}(2)$ vector-valued matrix $^{8}$

$$
\langle\hat{\mathbf{S}}\rangle_{\mathbf{k}}=\left(\begin{array}{cc}
\left\langle\Psi_{\mathbf{k}}^{+}|\hat{\mathbf{S}}| \Psi_{\mathbf{k}}^{+}\right\rangle & \left\langle\Psi_{\mathbf{k}}^{+}|\hat{\mathbf{S}}| \Psi_{\mathbf{k}}^{-}\right\rangle \\
\left\langle\Psi_{\mathbf{k}}^{-}|\hat{\mathbf{S}}| \Psi_{\mathbf{k}}^{+}\right\rangle & \left\langle\Psi_{\mathbf{k}}^{-}|\hat{\mathbf{S}}| \Psi_{\mathbf{k}}^{-}\right\rangle
\end{array}\right)
$$

in the subspace of the two degenerate states. Then the first diagonal element of such a matrix is aligned along the $z$ direction in gauge I. However, this causes inconsistencies in the extreme case of the so-called spin hot spots, ${ }^{16}$ when $\left\langle\Psi_{\mathbf{k}}^{+}\left|\hat{S}_{z}\right| \Psi_{\mathbf{k}}^{+}\right\rangle=-\left\langle\Psi_{\mathbf{k}}^{-}\left|\hat{S}_{z}\right| \Psi_{\mathbf{k}}^{-}\right\rangle$vanishes within this gauge.

First of all, the task is to identify conditions for which such a situation occurs. We can expect the spin mixing to be enhanced in the vicinity of accidental degeneracies in the BZ. ${ }^{15,16}$ For a Hamiltonian that depends on a set of parameters one can ask how many of these parameters need to be varied in order to encounter a twofold degenerate eigenvalue. This number is called codimension. According to a theorem by von Neumann and Wigner, ${ }^{17}$ for a generic Hamiltonian the answer is three. In other words, degeneracies are points in a three-dimensional parameter space such as the BZ. However, as we have already discussed above, in the case of a nonmagnetic crystal with inversion symmetry each band is twofold degenerate. As a consequence, accidental degeneracies of two different bands would have to be fourfold. The codimension of an intersection of four bands is five $\mathrm{e}^{18-20}$ and therefore the occurrence of an accidental degeneracy is infinitely unlikely in the BZ. This is the reason why only avoided crossings are practically observed
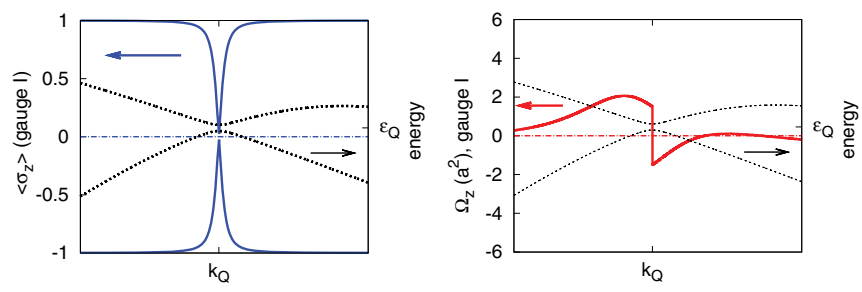

FIG. 1. (Color online) Left: $z$ component of the diagonal elements of the $\mathfrak{s u}(2)$ spin polarization (solid line) of the lower Kramers doublet within gauge I. Right: $z$ component of the first diagonal element of the corresponding $\mathfrak{s u}(2)$ Berry curvature (solid line) jumps in gauge I.

for nonmagnetic crystals with inversion symmetry. When two Kramers doublets get close at some point in the BZ they repel each other depending on the strength of the spin-orbit interaction. ${ }^{4}$ A simple model for such an avoided crossing was proposed and investigated by Scharf et al. ${ }^{18}$

Such a situation is illustrated in Fig. 1, where we present the results of a tight-binding calculation ${ }^{11}$ for a typical situation with spin-orbit driven avoided crossing of two bands. Each of them is a Kramers doublet. One can see that $\left\langle\Psi_{\mathrm{I}}^{+}\left|\hat{S}_{z}\right| \Psi_{\mathrm{I}}^{+}\right\rangle$ and $\left\langle\Psi_{\mathrm{I}}^{-}\left|\hat{S}_{z}\right| \Psi_{\mathrm{I}}^{-}\right\rangle$, calculated for the lower doublet and shown in the left panel of Fig. 1, vanish at the avoided crossing. In fact, the picture suggests that the spin polarizations of both states change sign, which means that by enforcing gauge I we move from the first to the second Kramers state going through the anticrossing point. Indeed, this is verified by the graph for the element $\Omega_{++}^{z}(\mathbf{k})$ of the corresponding Berry curvature presented in Fig. 1 (right panel) within the same gauge. Exactly at the point where the spin polarization vanishes $\Omega_{++}^{z}(\mathbf{k})$ changes sign, jumping from one degenerate state to the other. This jump is unsatisfactory, since the single-subband picture breaks down. A way out can be provided by a different gauge.

This alternative gauge describes the nonmagnetic crystal as the limit of vanishing exchange splitting in a corresponding magnetic crystal. Our task is then to find a unitary transformation that diagonalizes the perturbation operator $\hat{H}_{\mathrm{ex}} \sim \hat{S}_{z} \cdot B_{z}$ in the degenerate subspace of the Kramers doublet. In addition, we can label the two degenerate states using the same way as was chosen for gauge I. Therefore, this gauge, let us call it gauge II, requires the following conditions:

$$
\begin{gathered}
\left\langle\Psi_{\text {II }}^{+}\left|\mathcal{H}_{\text {ex }}\right| \Psi_{\text {II }}^{-}\right\rangle \sim\left\langle\Psi_{\text {II }}^{+}\left|\hat{S}_{z}\right| \Psi_{\text {II }}^{-}\right\rangle=0, \\
\left\langle\Psi_{\text {II }}^{+}\left|\hat{S}_{z}\right| \Psi_{\text {II }}^{+}\right\rangle=-\left\langle\Psi_{\text {II }}^{-}\left|\hat{S}_{z}\right| \Psi_{\text {II }}^{-}\right\rangle \geqslant 0 .
\end{gathered}
$$

Similar to gauge I, described in detail in Ref. 15, the above equation accounts again for the two free parameters one has to fix. In the nonrelativistic limit both gauges are identical. However, the spin-orbit coupling leads to finite $\left\langle\Psi_{\mathbf{k}}^{+}\left|\hat{S}_{x}\right| \Psi_{\mathbf{k}}^{+}\right\rangle=$ $-\left\langle\Psi_{\mathbf{k}}^{-}\left|\hat{S}_{x}\right| \Psi_{\mathbf{k}}^{-}\right\rangle$and $\left\langle\Psi_{\mathbf{k}}^{+}\left|\hat{S}_{y}\right| \Psi_{\mathbf{k}}^{+}\right\rangle=-\left\langle\Psi_{\mathbf{k}}^{-}\left|\hat{S}_{y}\right| \Psi_{\mathbf{k}}^{-}\right\rangle$if gauge II is imposed.

Let us discuss the linear transformation corresponding to this gauge in detail. We start with two arbitrary degenerate states $\Psi_{1}$ and $\Psi_{2}$ which are normalized and orthogonal to each other. For the sake of simplicity, here we omit the crystal momentum index. Now we are going to construct two new states $\Psi_{\text {II }}^{+}$and $\Psi_{\text {II }}^{-}$. One can write

$$
\Psi_{\mathrm{II}}^{+}=c_{1} \Psi_{1}+c_{2} \Psi_{2} \quad \text { and } \quad \Psi_{\mathrm{II}}^{-}=-c_{2}^{*} \Psi_{1}+c_{1}^{*} \Psi_{2},
$$


with $\left|c_{1}\right|^{2}+\left|c_{2}\right|^{2}=1$. Thus, the orthogonality and the normalization of $\Psi_{\text {II }}^{+}$and $\Psi_{\text {II }}^{-}$states are fulfilled. Then, the first line of Eq. (7) gives us

$$
2 c_{1} c_{2}\left\langle\Psi_{1}\left|\hat{S}_{z}\right| \Psi_{1}\right\rangle+c_{2}^{2}\left\langle\Psi_{1}\left|\hat{S}_{z}\right| \Psi_{2}\right\rangle-c_{1}^{2}\left\langle\Psi_{2}\left|\hat{S}_{z}\right| \Psi_{1}\right\rangle=0 .
$$

As solutions we have $c_{2} / c_{1}=x$ with

$$
x_{ \pm}=-\frac{\left\langle\Psi_{1}\left|\hat{S}_{z}\right| \Psi_{1}\right\rangle}{\left\langle\Psi_{1}\left|\hat{S}_{z}\right| \Psi_{2}\right\rangle} \pm \frac{\sqrt{\left(\left\langle\Psi_{1}\left|\hat{S}_{z}\right| \Psi_{1}\right\rangle\right)^{2}+\left|\left\langle\Psi_{1}\left|\hat{S}_{z}\right| \Psi_{2}\right\rangle\right|^{2}}}{\left\langle\Psi_{1}\left|\hat{S}_{z}\right| \Psi_{2}\right\rangle} .
$$

Here the sign should be chosen to fulfill the last condition of Eq. (7). In practice, one can always use, for instance, "+" during the transformation and sort all the new states to $\Psi_{\mathrm{II}}^{+}$and $\Psi_{\text {II }}^{-}$afterwards. Finally, using the normalization condition, we obtain

$$
c_{1}=1 / \sqrt{1+|x|^{2}} \text { and } c_{2}=x / \sqrt{1+|x|^{2}},
$$

where we have fixed a remaining arbitrary phase by the condition $c_{1}=\left|c_{1}\right|$.

In general, any gauge transformation can be described by the unitary matrix $\hat{U}$ as

$$
\left(\begin{array}{l}
\Psi_{1} \\
\Psi_{2}
\end{array}\right) \rightarrow\left(\begin{array}{l}
\Psi^{+} \\
\Psi^{-}
\end{array}\right)=\hat{U}\left(\begin{array}{l}
\Psi_{1} \\
\Psi_{2}
\end{array}\right) .
$$

Accordingly, the transformation of the spin polarization matrix in Eq. (6) is given by $\langle\hat{\mathbf{S}}\rangle_{\mathbf{k}} \rightarrow\left(\hat{U}^{T}\right)^{\dagger}\langle\hat{\mathbf{S}}\rangle_{\mathbf{k}}\left(\hat{U}^{T}\right)$, where the superscript $T$ denotes the transpose. Then, it is evident that $\operatorname{det}\left|\left\langle\hat{S}_{z}\right\rangle_{\mathbf{k}}\right|=-\left|\left\langle\Psi_{\mathbf{k}}^{+}\left|\hat{S}_{z}\right| \Psi_{\mathbf{k}}^{+}\right\rangle\right|^{2}-\left|\left\langle\Psi_{\mathbf{k}}^{+}\left|\hat{S}_{z}\right| \Psi_{\mathbf{k}}^{-}\right\rangle\right|^{2}$ should be gauge invariant. The related matrix can be also represented by a linear combination of the Pauli matrices: $\left\langle\hat{S}_{z}\right\rangle_{\mathbf{k}}=$ $a_{x}(\mathbf{k}) \hat{\sigma}_{x}+a_{y}(\mathbf{k}) \hat{\sigma}_{y}+a_{z}(\mathbf{k}) \hat{\sigma}_{z}$. Then, the unitary transformation $\hat{U}$ corresponds to a rotation of the real $\mathbf{k}$-dependent vector $\mathbf{a}=\left(a_{x}, a_{y}, a_{z}\right)$ in the three-dimensional Euclidean space. Its absolute value is also invariant, since one can easily obtain the identity $\operatorname{det}\left|\left\langle\hat{S}_{z}\right\rangle_{\mathbf{k}}\right|=-|\mathbf{a}|^{2}$. The conditions of gauge II, given by Eq. (7), imply $a_{x}=a_{y}=0$ and $a_{z}>0$. Hence, $\left\langle\Psi_{\text {II }}^{+}\left|\hat{S}_{z}\right| \Psi_{\text {II }}^{+}\right\rangle=a_{z}=|\mathbf{a}|$ assumes its maximum value with respect to all possible gauges. Consequently, if $\left\langle\Psi_{\mathbf{k}}^{+}\left|\hat{S}_{z}\right| \Psi_{\mathbf{k}}^{+}\right\rangle$ vanishes within gauge II, this quantity must be zero in any other gauge. Thus, gauge II should be the most appropriate gauge for discussions of the spin hot spots, since spin mixing is minimized and $\mathbf{k}$ points with $\left\langle\Psi_{\mathbf{k}}^{+}\left|\hat{S}_{z}\right| \Psi_{\mathbf{k}}^{+}\right\rangle=\left\langle\Psi_{\mathbf{k}}^{-}\left|\hat{S}_{z}\right| \Psi_{\mathbf{k}}^{-}\right\rangle=0$ as an artifact of a certain gauge are avoided.

Let us come back to the situation with the spin polarization and the Berry curvature shown in Fig. 1. We have performed similar calculations using gauge II, which are presented in Fig. 2. One can see that now the spin polarization remains finite and the Berry curvature is continuous. Thus, the ambiguity discussed above is resolved. Moreover, in contrast to Fig. 1, the peak of the Berry curvature is located now exactly at the avoided crossing, as could be expected intuitively.

We emphasize that within gauge II the diagonal elements of the vector-valued matrix $\langle\hat{\mathbf{S}}\rangle_{\mathbf{k}}$ given by Eq. (6) represent the analytical continuation of the magnetic spin polarizations for vanishing exchange splitting. Hence, gauge II should reflect the case of electrons injected from a ferromagnet or the presence of a weak magnetic field better than gauge I. In fact, such a gauge transformation is known to be used in the literature. ${ }^{16}$
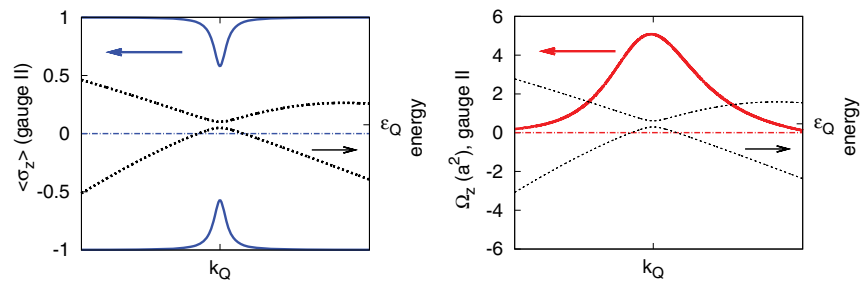

FIG. 2. (Color online) Left: $z$ component of the diagonal elements of the $\mathfrak{s u}(2)$ spin polarization (solid line) of the lower Kramers doublet in gauge II. Right: $z$ component of the first diagonal element of the corresponding $\mathfrak{s u}(2)$ Berry curvature (solid line) remains continuous within gauge II.

However, a detailed analysis of the advantages of this gauge for practical calculations was missing.

Let us consider a typical influence of the choice of gauge on some results obtained within the single-subband approximation. They are shown in Tables I and II for the spin relaxation time and the skew-scattering contribution to the spin Hall angle, respectively, which are calculated by the relativistic KKR method for both gauges. The related physical phenomena are caused by scattering at substitutional impurities, and all details of the calculations are described in Refs. 10 and 12 . Obviously, the difference between the two gauges should be larger the stronger the spin-orbit interaction is, since in the nonrelativistic limit they are identical. Indeed, this situation is well illustrated by the two tables. The maximal deviation of the presented values is of the order of $0.1 \%, 1 \%$, and $10 \%$ for $\mathrm{Cu}, \mathrm{Au}$, and $\mathrm{Pt}$, respectively. In fact, the numerical accuracy in the corresponding calculations is $\sim 1 \%$. Therefore, the gauge choice can be important for materials such as Pt, where the spin-orbit interaction is strongly enhanced. ${ }^{15}$

Above we have shown that gauge II solves the technical problems arising when one needs to illustrate the non-Abelian Berry curvature within the single-subband approximation. Another advantage of such a gauge transformation is the calculation of the intrinsic spin Hall conductivity (SHC) for nonmagnetic crystals with a center of inversion. A simplified, but still a complete non-Abelian, semiclassical treatment, based on Ref. 8, provides the following expression for the intrinsic SHC:

$$
\sigma_{x y}^{z}=\frac{e^{2}}{2 \hbar} \int_{\mathrm{BZ}} \frac{d \mathbf{k}}{(2 \pi)^{3}} f_{0}\left(E_{F}, \mathbf{k}\right) \operatorname{Tr}\left[\left\langle\hat{S}^{z}\right\rangle_{\mathbf{k}}\left\langle\hat{\Omega}^{z}\right\rangle_{\mathbf{k}}\right] .
$$

TABLE I. Numerical results for the spin relaxation time (in ps), for both gauges using the single-subband approximation, for $\mathrm{Cu}, \mathrm{Au}$, and Pt bulk crystals with different impurities.

$$
\mathrm{Cu} \quad \mathrm{Au} \quad \mathrm{Pt}
$$

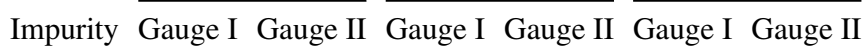

\begin{tabular}{lcccccc}
\hline $\mathrm{Li}$ & 18.5 & 18.5 & 0.36 & 0.37 & 0.15 & 0.16 \\
$\mathrm{C}$ & 4.29 & 4.29 & 0.13 & 0.13 & 0.05 & 0.06 \\
$\mathrm{~N}$ & 4.96 & 4.96 & 0.13 & 0.13 & 0.04 & 0.04 \\
$\mathrm{Mg}$ & 14.6 & 14.6 & 0.28 & 0.28 & 0.16 & 0.18 \\
$\mathrm{Ag}$ & 13.0 & 13.0 & 1.02 & 1.03 & 0.24 & 0.27 \\
$\mathrm{Bi}$ & 0.12 & 0.12 & 0.10 & 0.10 & 0.07 & 0.07 \\
\hline \hline
\end{tabular}


TABLE II. Skew-scattering contribution to the spin Hall angle for $\mathrm{Cu}, \mathrm{Au}$, and Pt bulk crystals with different impurities.

\begin{tabular}{|c|c|c|c|c|c|c|}
\hline \multirow[b]{2}{*}{ Impurity } & \multicolumn{2}{|c|}{$\mathrm{Cu}$} & \multicolumn{2}{|c|}{$\mathrm{Au}$} & \multicolumn{2}{|c|}{$\mathrm{Pt}$} \\
\hline & Gauge I & Gauge II & Gauge I & Gauge II & Gauge I & Gauge II \\
\hline $\mathrm{Li}$ & 2.3 & 2.3 & 7.2 & 7.4 & -2.3 & -2.3 \\
\hline $\mathrm{C}$ & 6.6 & 6.6 & 96.0 & 97.0 & -2.8 & -1.0 \\
\hline $\mathrm{N}$ & 7.0 & 7.0 & 64.0 & 65.0 & 11.0 & 13.0 \\
\hline $\mathrm{Mg}$ & -1.5 & -1.5 & -8.2 & -8.3 & -3.8 & -4.1 \\
\hline $\mathrm{Ag}$ & 0.26 & 0.26 & 4.8 & 4.9 & -2.7 & -2.9 \\
\hline $\mathrm{Bi}$ & 81.0 & 81.0 & 14.0 & 15.0 & -1.2 & -1.6 \\
\hline
\end{tabular}

Here the Fermi function $f_{0}\left(E_{F}, \mathbf{k}\right)$ restricts the integral over the BZ to the occupied states. Clearly, this SHC is a gauge independent quantity. However, with an arbitrary gauge it is necessary to know all four components of both $\left\langle\hat{S}^{z}\right\rangle_{\mathbf{k}}$ and $\left\langle\hat{\Omega}^{z}\right\rangle_{\mathbf{k}}$ Hermitian matrices. This means that one should calculate at least the four independent quantities $\hat{S}_{++}^{z}, \hat{S}_{+-}^{z}, \hat{\Omega}_{++}^{z}$, and $\hat{\Omega}_{+-}^{z}$. Within gauge II, the problem is reduced to the computation of two real quantities $\hat{S}_{++}^{z}$ and $\hat{\Omega}_{++}^{z}$. Such an approach was successfully applied in Ref. 21 for calculation of the intrinsic SHC for bulk Au and Pt.

In summary, the influence of the chosen gauge on the results obtained within the single-subband approximation is discussed for nonmagnetic and inversion symmetric crystals. We show that the gauge corresponding to the analytical continuation of the magnetic case with vanishing exchange splitting is particularly appropriate for calculations based on the discussed approximation. Especially, it provides a suitable way to deal with the Berry curvature playing a crucial role in the modern semiclassical theory. In addition, this gauge can minimize the effort of numerical calculations within the non-Abelian approach.

This work was supported by the International Max Planck Research School for Science and Technology of Nanostructures and by the Deutsche Forschungsgemeinschaft (DFG) via SFB 762. One of us (B.L.G.) would like to thank Michael V. Berry for enlightening discussions on the role of degeneracy of Kramers doublets. In addition, M.G. acknowledges financial support from the DFG via a research fellowship (GR3838/1-1).

*falko.pientka@fu-berlin.de

${ }^{1}$ H. A. Kramers, Proceedings Royal Acad. Amsterdam 33, 959 (1930).

${ }^{2}$ R. J. Elliott, Phys. Rev. 96, 266 (1954).

${ }^{3}$ M. V. Berry, Proc. R. Soc. London, Ser. A 392, 45 (1984).

${ }^{4}$ A. Bohm, A. Mostafazadeh, H. Koizumi, Q. Niu, and J. Zwanziger, The Geometric Phase in Quantum Systems (Springer, Berlin, 2003).

${ }^{5}$ G. Sundaram and Q. Niu, Phys. Rev. B 59, 14915 (1999).

${ }^{6}$ M.-C. Chang and Q. Niu, J. Phys.: Condens. Matter 10, 193202 (2008).

${ }^{7}$ F. Wilczek and A. Zee, Phys. Rev. Lett. 52, 2111 (1984).

${ }^{8}$ R. Shindou and K.-I. Imura, Nucl. Phys. B 720, 399 (2005).

${ }^{9}$ S. Lowitzer, M. Gradhand, D. Ködderitzsch, D. V. Fedorov, I. Mertig, and H. Ebert, Phys. Rev. Lett. 106, 056601 (2011).

${ }^{10}$ M. Gradhand, D. V. Fedorov, P. Zahn, and I. Mertig, Phys. Rev. B 81, 020403(R) (2010).

${ }^{11}$ F. Pientka, Diploma thesis, University Halle, 2010.

${ }^{12}$ M. Gradhand, D. V. Fedorov, P. Zahn, and I. Mertig, Phys. Rev. Lett. 104, 186403 (2010).

${ }^{13}$ M. Gradhand, D. V. Fedorov, P. Zahn, and I. Mertig, Phys. Rev. B 81, 245109 (2010).

${ }^{14}$ To highlight a special choice of the wave functions sometimes we put a gauge index instead of the omitted index $\mathbf{k}$.

${ }^{15}$ M. Gradhand, M. Czerner, D. V. Fedorov, P. Zahn, B. Yu. Yavorsky, L. Szunyogh, and I. Mertig, Phys. Rev. B 80, 224413 (2009).

${ }^{16}$ J. Fabian and S. Das Sarma, Phys. Rev. Lett. 81, 5624 (1998).

${ }^{17}$ J. von Neumann and E. P. Wigner, Phys. Z. 30, 467 (1929).

${ }^{18}$ R. Scharf, B. Dietz, M. Kus, F. Haake, and M. V. Berry, Europhys. Lett. 5, 383 (1988).

${ }^{19}$ J. E. Avron, L. Sadun, J. Segert, and B. Simon, Phys. Rev. Lett. 61, 1329 (1988).

${ }^{20}$ Y. Hatsugai, New J. Phys. 12, 065004 (2010).

${ }^{21}$ M. Gradhand, D. V. Fedorov, F. Pientka, P. Zahn, I. Mertig, and B. L. Györffy, Phys. Rev. B 84, 075113 (2011). 